\title{
Simulation of portal/hepatic vein associated remnant liver ischemia/congestion by three-dimensional visualization technology based on preoperative CT scan
}

\author{
Xiao-Long $\mathrm{Li}^{1,2 \#}$, Bin $\mathrm{Xu}^{1,2 \#}$, Xiao-Dong $\mathrm{Zhu}^{1,2 \#}$, Cheng Huang ${ }^{1,2}$, Guo-Ming Shi, ${ }^{1,2}$, Ying-Hao Shen ${ }^{1,2}$, \\ Dong $\mathrm{Wu}^{3}$, Min Tang ${ }^{3}$, Zhao-You Tang ${ }^{1,2}$, Jian Zhou ${ }^{1,2}$, Jia Fan ${ }^{1,2}$, Hui-Chuan Sun ${ }^{1,2}$ \\ ${ }^{1}$ Department of Liver Surgery and Transplantation, Liver Cancer Institute, Zhongshan Hospital, Fudan University, Shanghai, China; ${ }^{2}$ Key \\ Laboratory of Carcinogenesis and Cancer Invasion of Ministry of Education, Shanghai, China; ${ }^{3}$ Department of Radiology, Zhongshan Hospital, \\ Fudan University, Shanghai, China \\ Contributions: (I) Conception and design: XL Li, B Xu, XD Zhu, HC Sun; (II) Administrative support: ZY Tang, J Zhou, J Fan; (III) Provision \\ of study materials or patients: XL Li, B Xu, XD Zhu, HC Sun; (IV) Collection and assembly of data: XL Li, B Xu, XD Zhu, C Huang, GM Shi, \\ YH Shen, D Wu, M Tang; (V) Data analysis and interpretation: XL Li, B Xu, XD Zhu, HC Sun; (VI) Manuscript writing: All authors; (VII) Final \\ approval of manuscript: All authors. \\ \#These authors contributed equally to this work. \\ Correspondence to: Hui-Chuan Sun. Department of Liver Surgery and Transplantation, Liver Cancer Institute, Zhongshan Hospital, Fudan University, \\ 180 Fenglin Road, Shanghai 200032, China. Email: sun.huichuan@zs-hospital.sh.cn.
}

Background: Remnant liver hypoperfusion is frequently observed after hepatectomy, and associated with a higher risk of postoperative complications and poorer survival. However, the development of remnant liver hypoperfusion was not fully understood.

Methods: We retrospectively analyzed patients who received hepatectomy and took contrast-enhanced computed tomography (CT) scans before, 1-week (POW1) and 4-week (POW4) after resection in our department from June 2017 to July 2019. We simulated and estimated the occurrence of portal-vein-related remnant liver ischemia (RLI) and hepatic-vein-related remnant liver congestion (RLC) after hepatectomy via three-dimensional visualization technology (3DVT) according to blood vessels ligated in the resection; then we analyzed association between the estimated RLI, RLC, and postoperative clinical outcomes.

Results: A total of 102 eligible patients were analyzed. Remnant liver hypoperfusion was observed in 47 (46\%) patients in the POW1 CT scans and shrunk in the POW4 CT scans. RLC had better diagnostic significance than RLI in predicting remnant liver hypoperfusion [area under receiver operating characteristic (ROC) curve: 0.745 vs. $0.569, \mathrm{P}=0.026$ ]. Multivariate analysis showed that larger RLI [odds ratio (OR), 1.154; 95\% confidence interval (CI), 1.075-1.240; $\mathrm{P}<0.001]$ was independent risk factor for post-hepatectomy liver failure (PHLF). Besides, larger RLC (OR, 1.114; 95\% CI, 1.032-1.204; P=0.006) was independent risk factor for major postoperative complications.

Conclusions: Remnant liver hypoperfusion can be predicted during the preoperative surgical plan by 3DVT. Portal vein related RLI was associated with PHLF, and hepatic vein related RLC was associated with major postoperative complications. Preservation of the hepatic vein and complete removal of the perfusion territory of ligated vessels are essential procedures to reduce RLI/RLC and the risk of PHLF or other surgical complications.

Keywords: Hepatectomy; three-dimensional visualization technology (3DVT); remnant liver ischemia/congestion (RLI/RLC); post-hepatectomy liver failure (PHLF); postoperative complication

Submitted Dec 10, 2020. Accepted for publication Mar 12, 2021.

doi: $10.21037 /$ atm-20-7920

View this article at: http://dx.doi.org/10.21037/atm-20-7920 


\section{Introduction}

Hepatectomy is the treatment mainstay for liver tumor. Although hepatectomy has become much safer than it had been, it is still associated with a high risk of morbidity and mortality, mostly related to post-hepatectomy liver failure (PHLF) and postoperative complications (1-3).

Localized ischemia or congested area after hepatectomy can be found because of unintentional damage to portal vein or hepatic vein responsible for the remnant liver, even after anatomical resection $(4,5)$. Prior research has shown that patients with remnant liver ischemia (RLI), defined as partial liver hypoperfusion presented on postoperative computed tomography (CT) scans, had a higher risk of biliary leak and higher peak levels of postoperative serum aspartate aminotransferase (AST) and alanine aminotransferase (ALT) (6). Remnant liver hypoperfusion was also associated with a negative oncological outcome in patients with malignant tumors $(7,8)$. However, postoperative CT scans cannot distinguish outflow-injured congestion from inflowinjured ischemia in liver parenchyma because they had similar radiologic images $(9,10)$.

Three-dimensional visualization technology (3DVT) was first introduced in the year 2000 and has become a popular tool to visualize intrahepatic structure and guide precise liver resection (11-13). Virtual hepatectomy based on 3DVT also allows patients with hepatocellular carcinoma (HCC) and impaired liver function or advanced colorectal liver metastases (CLRM) to undergo radical hepatectomy without compromising long-term outcomes (14). Apart from visualization of the intrahepatic vessels, 3DVT offers a great advantage over conventional preoperative evaluation by calculating the liver volumes supported by a single vessel (either portal vein or hepatic vein) (15), so preoperative simulation of RLI or remnant liver congestion (RLC) is feasible once a transection plane is determined on the virtual hepatectomy. We therefore conducted a retrospective study to evaluate (I) the feasibility of RLI/RLC assessment by $3 \mathrm{DVT}$, (II) association between estimated RLI/RLC and remnant liver hypoperfusion on postoperative week (POW) 1 and POW4 CT scans, and (III) explore the association between estimated RLI/RLC and post-hepatectomy outcomes.

We present the following article in accordance with the STARD reporting checklist (available at http://dx.doi. org/10.21037/atm-20-7920).

\section{Methods}

\section{Patients}

All patients received surgical resection in our department from June 2017 to July 2019 formed a convenience series; the inclusion criteria were as follows: (I) patients received hepatectomy for liver tumor; (II) contrast-enhanced CT scans were conducted before, 1 week and 4 weeks after resection; and (III) blood routine test, biochemical test, coagulatory function test, and liver stiffness (LS) assessed by shear wave elastography were conducted within one week prior to surgery. The exclusion criteria included: (I) patients who received portal vein embolization (PVE) or associating liver partition and portal vein ligation for staged hepatectomy (ALPPS); (II) patients with missing data. The study was conducted in accordance with the Declaration of Helsinki (as revised in 2013). The study was approved by institutional ethics board of Zhongshan Hospital, Fudan University (No. B2018-254) and informed consent was taken from all individual participants.

\section{Hepatectomy and postoperative management}

Parenchyma transection was conducted by alternating use of ultrasonic dissector (Harmonic) and clamp-crushing technique. Complete hemostasis was achieved by ligation or electrocoagulation. The Pringle maneuver or hepatic vein occlusion was used to control bleeding from inflow or outflow vessels if necessary. The volume of resected specimen was measured by Archimedes water-displacement method.

All patients received routine management after operation (16). Blood routine test, liver function, renal function, and coagulatory function test were monitored every 2 days. Patients received supportive treatments, including fluid infusion with supplementation of glucose, albumin (ALB) transfusion if the serum ALB level was $<35 \mathrm{~g} / \mathrm{L}$, oral anti-hepatitis B virus (HBV) therapy if serum HBV-DNA was detectable, intravenous administration of ademetionine butanedisulfonate if serum bilirubin was $>3 \times$ ULN, plasma or coagulative reagent transfusion if prothrombin time (PT) was extended, and management of postoperative complications.

Remnant liver hypoperfusion was measured on the POW1 and POW4 CT scans, and was defined as reduced or absent contrast enhancement in the portal phase, 
as previous reports $(6,7)$. In this study, three principle surgeons who conducted liver resection for patients and one radiologist evaluated and measured remnant liver hypoperfusion respectively. PHLF was defined as postoperative deterioration of liver function with an increase in the INR and concomitant hyperbilirubinemia on or after postoperative day 5 as proposed by the International Study Group of Liver Surgery (ISGLS) (3). Postoperative complications were recorded and categorized according to the modified Clavien-Dindo classification (17).

\section{Process of $3 D$ reconstruction}

Contrast-enhanced multiphase CT scans were obtained using a 320-section scanner (Aquilion One; TOSHIBA, Japan). Arterial, portal venous, and delayed phases were obtained by the time of, adding $50-60 \mathrm{~s}$, and $80-85 \mathrm{~s}$ to the peak aortic enhancement, respectively. Scanning parameters were as follows: tube voltage, $120 \mathrm{kV}$; tube current, 50-250 mA; 512×512 matrix; pitch, 1.5; section thickness, $3 \mathrm{~mm}$; image reconstruction increment, $1 \mathrm{~mm}$.

The $3 \mathrm{D}$ reconstruction was performed with the abdominal CT image post-processing software (Shenzhen Yorktal Digital Medical Imaging Technology Co., Ltd., China) using the preoperative, POW1 and POW4 CT scan data, as follows: (I) import thin-layer CT scan DICOM image data (layer thickness of $1.00 \mathrm{~mm}$ ); (II) select multiple seed points in the target area in the $2 \mathrm{D}$ image, segment the liver semi-automatically by region growth algorithm and periodic iteration segmentation; (III) segment images of the hepatic artery, hepatic vein and portal vein automatically by threshold analysis algorithm; (IV) segment tumor and generate 3D model by interactive segmentation algorithm; and $(\mathrm{V})$ complete liver volume measurement by $3 \mathrm{D}$ volume measurement module.

\section{Liver volumetry and estimated $R L I / R L C$ on preoperative CT scan}

The estimated standardized liver volume (SLV) was calculated using Urata's formula (18). The volume of large vessels, including the inferior vena cava and the extrahepatic portal vein, dilatated biliary tract, the major fissures, and the gallbladder fossa were excluded in calculating liver volume (19). The perfusion territory affiliated to one selected vessel was calculated using an algorithm based on the Voronoi tessellation, which is bordered by a line that runs at an equal distance from the surrounding vessels (15).
The transection plane was retrospectively determined on the $3 \mathrm{D}$ image of the preoperative liver by the principle surgeons and radiologist referring to the resection margin and ligated vessels observed in the $3 \mathrm{D}$ image of the POW1 liver (Figure 1), then the estimated future remnant liver volume (eFRL) was calculated (Figure 2A,B). Vascular perfusion territory affiliated to the ligated portal veins or hepatic veins was also calculated. Remnant liver parenchyma without support of portal vein was defined as the estimated portal-vein-based RLI (eRLI) (Figure 2C,D). Similarly, remnant liver parenchyma without support of hepatic vein was defined as the estimated hepatic-vein-based RLC (eRLC) (Figure 2E,F).

To reduce individual variation, we used SLV as the denominator to standardize liver volume, and then obtained the eRLI/SLV, eRLC/SLV, and eFRL/SLV for further analysis (18).

\section{Statistical analysis}

Continuous variables were reported as mean [standard deviation (SD)] or median [interquartile range (IQR)], as appropriate. Categorical variables were reported as numbers and percentages, and compared using Pearson's $\chi^{2}$ analysis or Fisher's exact test. Repeated measures of remnant liver hypoperfusion on POW1 and POW4 were compared by Wilcoxon's Sign Rank Test. Predictive performance was assessed using the receiver operating characteristic (ROC) curve and compared by Delong's test. Univariate followed by multivariate logistic regression were used to evaluate the independent risk factors for PHLF and postoperative complications. All statistical tests were two-tailed, and a $\mathrm{P}$ value $<0.05$ indicated a significant difference. Statistical analyses were performed using SPSS Version 25.0 (IBM Corp., Armonk, NY, USA) and R version 3.5.2 (R Core Team, Vienna, Austria).

\section{Results}

\section{Demographic characteristics and liver volumetry}

One hundred and two patients (22 females and 80 males) were eligible for this study. The main indications for resection were HCC, intrahepatic cholangiocarcinoma (ICC) or hepatic hemangioma. Cirrhosis was found in $24(24 \%)$ patients by histological examination. All patients were Child-Pugh class A, and the mean LS measurements was $10.0 \pm 3.4 \mathrm{kPa}$ (Table 1). 
A

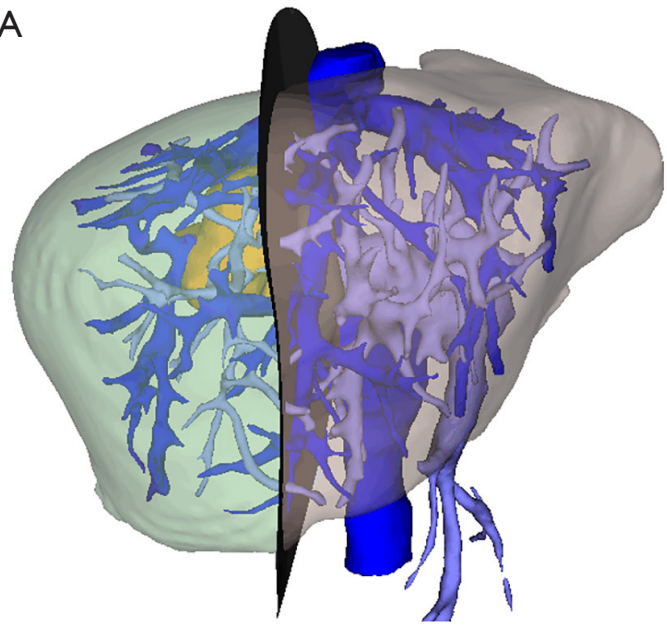

C

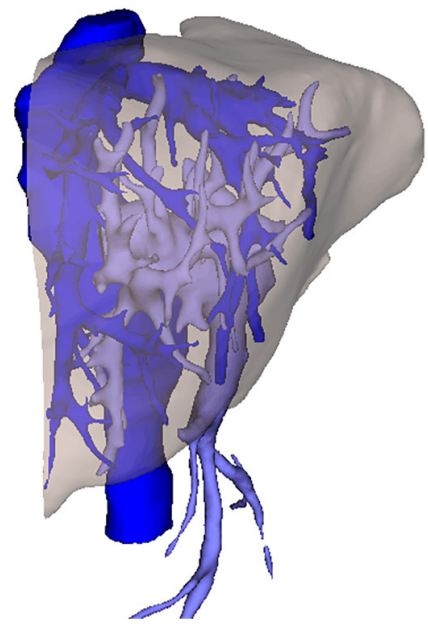

$\mathrm{E}$

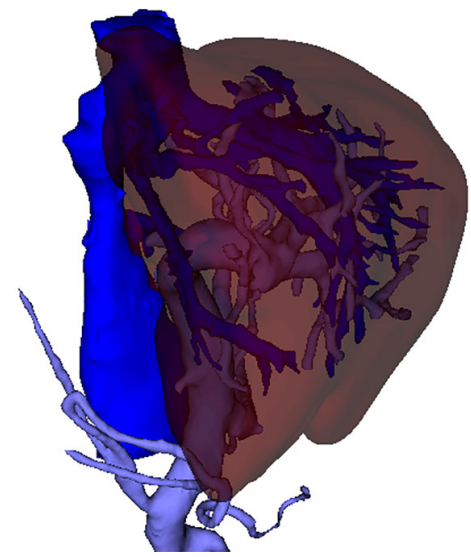

B
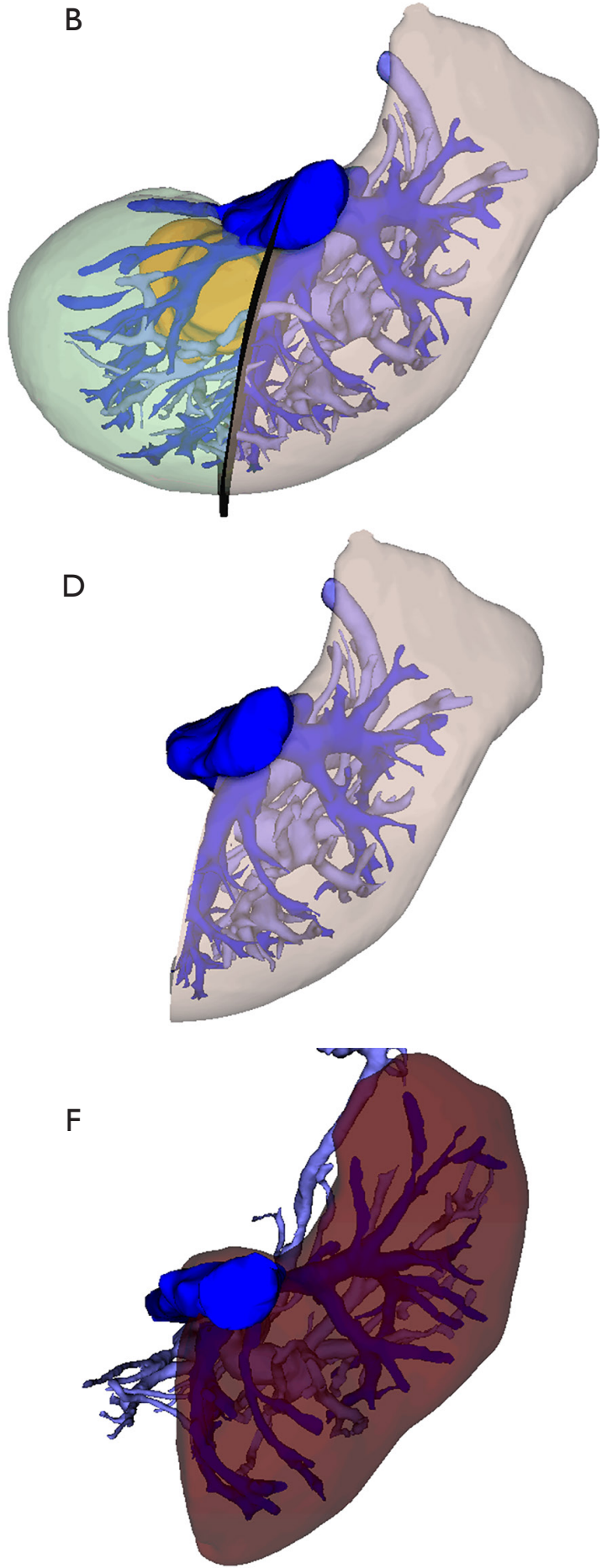

Figure 1 The retrospective determination of transection plane. The transection plane was retrospectively determined on the 3D image of the preoperative liver by the principle surgeons and radiologist referring to the resection margin and ligated vessels observed in the 3D image of the POW1 liver. (A,B) Determination of the transection on the 3D image of the preoperative liver. The dark blue area is inferior vena cava and hepatic veins, the light blue area is portal veins, the orange area is the tumor, and the gray curved surface is the transection plane. (C,D) The estimated remnant liver after virtual hepatectomy on the 3D image of the preoperative liver. (E,F) The actual remnant liver on the 3D image of the postoperative liver. 3D, three-dimensional; POW, postoperative week. 
A

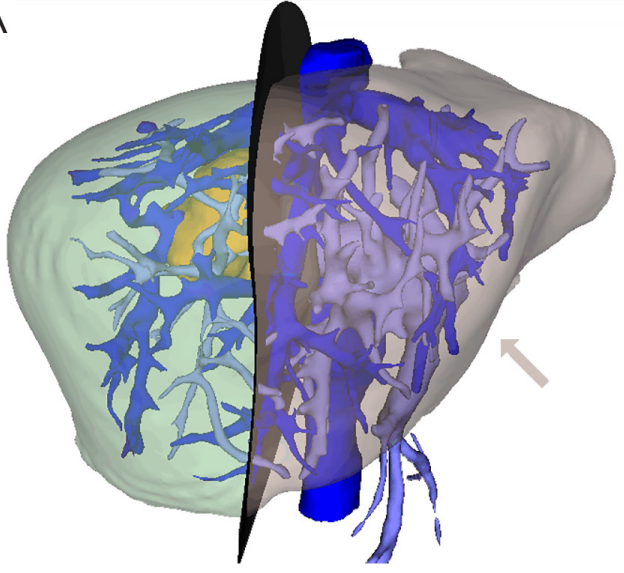

C
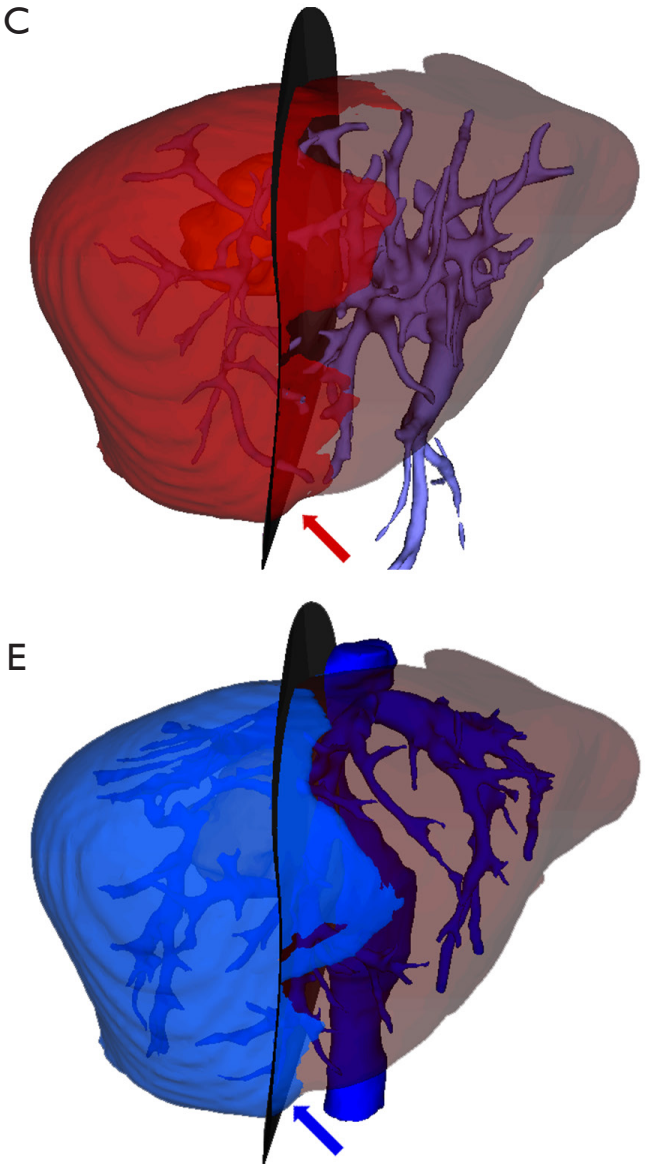
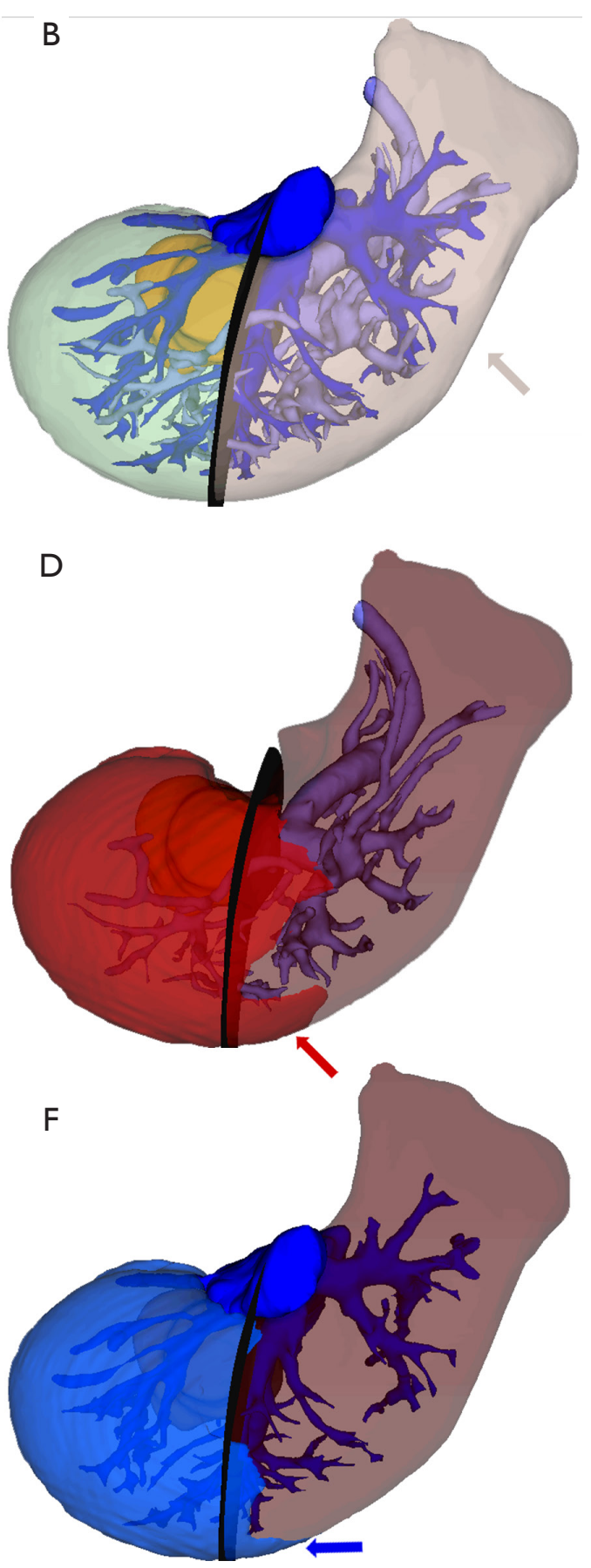

Figure 2 Calculation of estimated future remnant liver and RLI/RLC. (A,B) Determination of the transection plane and calculation of eFRL. The dark blue area is inferior vena cava and hepatic veins, the light blue area is portal veins, and the orange area is the tumor. The gray curved surface is the transection plane, and the light pink arrow is the eFRL. (C,D) The eRLI (red arrow) was calculated by subtracting the estimated resected liver from the perfusion territory (red area) of the ligated portal vein. The gray curved surface is the transection plane, and the orange area is the tumor. (E,F) The eRLC (blue arrow) was calculated by subtracting the estimated resected liver from the perfusion territory (blue area) of the ligated hepatic vein. The gray curved surface is the transection plane, and the orange area is the tumor. RLI, remnant liver ischemia; RLC, remnant liver congestion; eFRL, estimated future remnant liver volume; eRLI, estimated portal-veinassociated RLI; eRLC, estimated hepatic-vein-associated RLC. 
Table 1 Baseline characteristics, surgical procedure, liver volumetry and pathological examination results of all patients

\begin{tabular}{|c|c|}
\hline Variables & Values \\
\hline Age, mean [SD], years & $54.3[12.7]$ \\
\hline \multicolumn{2}{|l|}{ Sex, n [\%] } \\
\hline Female & 22 [22] \\
\hline Male & $80[78]$ \\
\hline \multicolumn{2}{|l|}{ Indications for resection, $\mathrm{n}$ [\%] } \\
\hline $\mathrm{HCC}$ & $57[56]$ \\
\hline ICC & $22[21]$ \\
\hline Hepatic hemangioma & 13 [13] \\
\hline Other malignant tumor & 2 [2] \\
\hline Other benign tumor & $8[8]$ \\
\hline Tumor diameter, mean [SD], cm & $8.9[4.6]$ \\
\hline \multicolumn{2}{|l|}{ Laboratory test results } \\
\hline $\mathrm{TB}$, median [IQR], $\mu \mathrm{mol} / \mathrm{L}$ & $12.6[9.8,15.2]$ \\
\hline ALB, mean [SD], g/L & $43.3[4.3]$ \\
\hline ALT, median [IQR], U/L & $25[17,46]$ \\
\hline AST, median [IQR], U/L & $31[20,50]$ \\
\hline GGT, median [IQR], U/L & $83[44,163]$ \\
\hline $\mathrm{PA}$, mean $[S D], \mathrm{g} / \mathrm{L}$ & $0.21[0.06]$ \\
\hline PT, mean [SD], s & $11.5[0.9]$ \\
\hline $\mathrm{Cr}$, mean $[\mathrm{SD}], \mu \mathrm{mol} / \mathrm{L}$ & $73.0[13.8]$ \\
\hline $\mathrm{HB}$, mean [SD], g/L & $138[19]$ \\
\hline PLT, median $[\mathrm{IQR}], \times 10^{9} / \mathrm{L}$ & $197[156,253]$ \\
\hline WBC, median $[I Q R], \times 10^{9} / \mathrm{L}$ & $5.9[5.0,7.4]$ \\
\hline \multicolumn{2}{|l|}{ HBsAg, n [\%] } \\
\hline+ & $58[57]$ \\
\hline- & $44[43]$ \\
\hline \multicolumn{2}{|l|}{ HBeAg, n [\%] } \\
\hline+ & $10[10]$ \\
\hline- & $92[90]$ \\
\hline \multicolumn{2}{|l|}{$\mathrm{CSPH}, \mathrm{n}[\%]$} \\
\hline Yes & $12[12]$ \\
\hline No & $90[88]$ \\
\hline LS, mean [SD], kPa & $10.0[3.4]$ \\
\hline \multicolumn{2}{|l|}{ Anatomical resection, $\mathrm{n}[\%]$} \\
\hline Yes & $46[45]$ \\
\hline No & 56 [55] \\
\hline
\end{tabular}

Table 1 (continued)
Table 1 (continued)

\begin{tabular}{|c|c|}
\hline Variables & Values \\
\hline Hilar occlusion, median [IQR], min & $20[16,26]$ \\
\hline Blood lose, median [IQR], mL & $400[200,600]$ \\
\hline \multicolumn{2}{|l|}{ Blood transfusion, $\mathrm{n}$ [\%] } \\
\hline Yes & $18[20]$ \\
\hline No & 84 [82] \\
\hline SLV, mean [SD], mL & $1,240.9[123.0]$ \\
\hline TLV, median [IQR], mL & $1,579.3[1,324.4,1,859.5]$ \\
\hline Tumor volume, median [IQR], mL & $180.4[62.8,554.0]$ \\
\hline eFRL/SLV, mean [SD], \% & $68.7[19.4]$ \\
\hline \multicolumn{2}{|l|}{ eRLI, n [\%] } \\
\hline Absent & 50 [49] \\
\hline Present & $52[51]$ \\
\hline eRLI/SLV, median [IQR], \% & $4.5[1.6,10.6]$ \\
\hline \multicolumn{2}{|l|}{ eRLC, n [\%] } \\
\hline Absent & $42[41]$ \\
\hline Present & 60 [59] \\
\hline eRLC/SLV, median [IQR], \% & $7.5[2.7,12.3]$ \\
\hline \multicolumn{2}{|l|}{ Inflammation grade, $\mathrm{n}$ [\%] } \\
\hline G0 & 36 [35] \\
\hline G1 & 19 [19] \\
\hline G2 & 40 [39] \\
\hline G3 & 7 [7] \\
\hline \multicolumn{2}{|l|}{ Fibrosis stage, $n$ [\%] } \\
\hline so & 36 [35] \\
\hline S1 & $8[8]$ \\
\hline $\mathrm{S} 2$ & 19 [19] \\
\hline S3 & 15 [15] \\
\hline S4 & 24 [23] \\
\hline
\end{tabular}

HCC, hepatocellular carcinoma; ICC, intrahepatic cholangiocarcinoma; TB, total bilirubin; ALB, albumin; ALT, alanine aminotransferase; AST, aspartate aminotransferase; GGT, $\gamma$-glutamyl transpeptidase; PA, pre-albumin; INR, international normalized ratio; $\mathrm{Cr}$, creatinine; $\mathrm{HB}$, hemoglobin; PLT, platelet count; WBC, white blood cell; HBsAg, hepatitis B s antigen; HBeAg, hepatitis $\mathrm{B}$ e antigen; $\mathrm{CSPH}$, clinical significant portal hypertension; LS, liver stiffness; SLV, standardized liver volume; TLV, total liver volume; eFRL, estimated future remnant liver volume; eRLI, estimated portal-vein-associated remnant liver ischemia; eRLC, estimated hepatic-vein-associated remnant liver congestion. 
Table 2 Abbreviations in this study

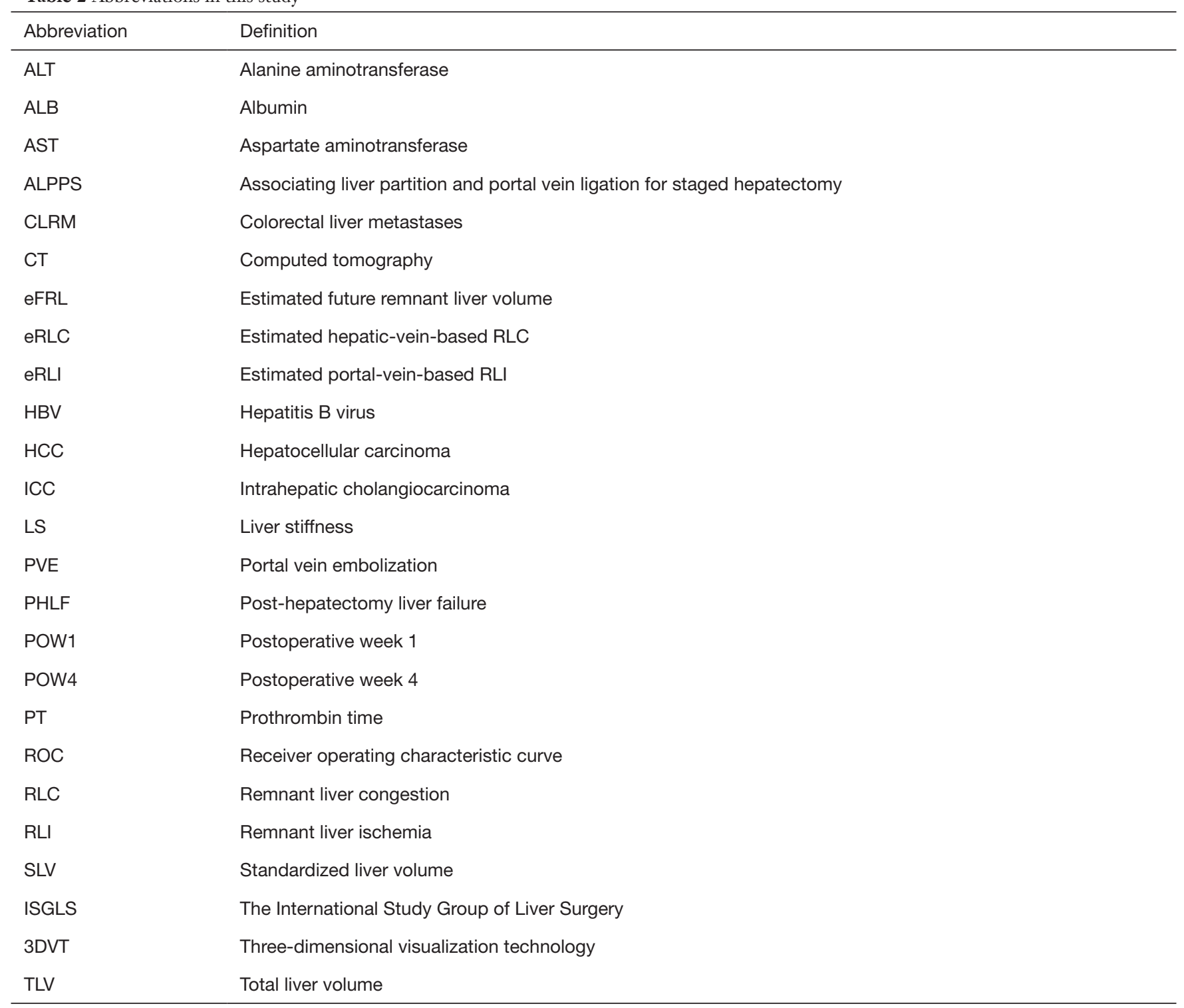

The types of hepatectomy are shown in Table 1. Fortysix patients (45\%) received anatomical resection (Table 1). The median hilar occlusion time was 20 minutes and median blood loss was $400 \mathrm{~mL}$; 18 (18\%) patients received blood transfusion during operation. The mean SLV was $1,240.9 \pm 123.0 \mathrm{~mL}$ and the median total liver volume (TLV) was $1,579.3(1,324.4,1,859.5) \mathrm{mL}$. The median tumor volume was $180.4(62.8,554.0) \mathrm{mL}$ (Table 2). A significant correlation existed between the predicted volume of resected liver and the volume of resected liver measured by Archimedes water-displacement method $\left(R^{2}=0.989\right.$,
$\mathrm{P}<0.0001$; Figure S1).

Postoperative complications occurred in 46 patients (45\%), including grade I in 26 , grade II in 5 , and grade III in 15 (10: subdiaphragmatic fluid collection; 5: pleural effusion requiring additional percutaneous drainage and antibiotic treatment). We categorized grade I and II as minor complication, and grade III and above as major complication, for further analysis. Thirty-six patients (35\%) exhibited PHLF, which was classified as grade A in 33 patients and grade B in 3 patients. There was no persistent PHLF or mortality within 3 months after hepatectomy. 


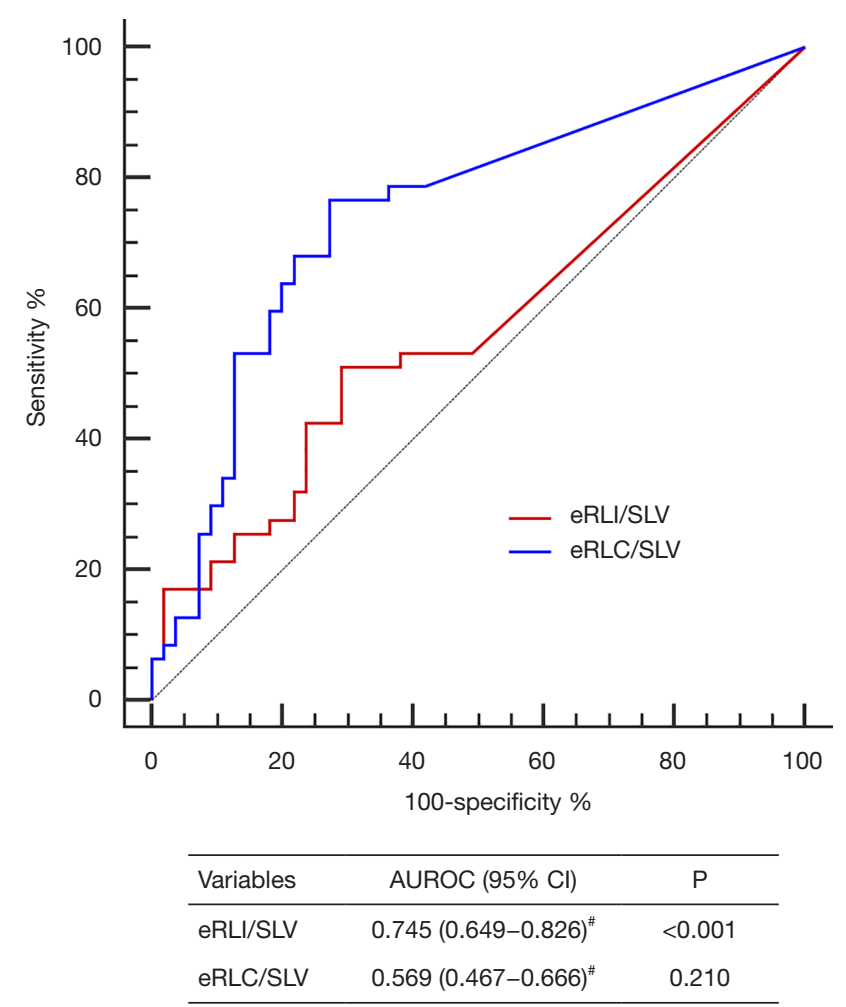

Figure 3 Prediction of remnant liver hypoperfusion detected on postoperative CT scans by estimated hepatic-vein-associated RLI or RLC. ROC analysis of the predictive ability of eRLI and eRLC for remnant liver hypoperfusion detected on postoperative CT scans. ", the predictive ability of eRLC/SLV was significantly better than that of eRLI/SLV ( $\mathrm{P}=0.026$, Delong's test). CT, computed tomography; RLI, remnant liver ischemia; RLC, remnant liver congestion; ROC, receiver operating characteristic; eRLI, estimated portal-vein-associated RLI; eRLC, estimated hepaticvein-associated RLC; SLV, standardized liver volume; AUROC, area under ROC curve.

\section{Incidence of remnant liver hypoperfusion}

Remnant liver hypoperfusion was observed in 47 (46\%) patients in the POW1 CT scans. Compared with patients without remnant liver hypoperfusion, those with remnant liver hypoperfusion were associated with higher preoperative white blood cell count $[6.3(5.5,8.0)$ vs. 5.5 (4.7, 7.4), $\mathrm{P}=0.032]$, higher percentage of non-anatomical resection $(68 \%$ vs. 44\%, $\mathrm{P}=0.013)$, and higher incidence of major postoperative complications ( $23 \%$ vs. $7 \%, \mathrm{P}=0.022)$. However, the incidence of PHLF between patients with or without remnant liver hypoperfusion were not different ( $43 \%$ vs. $29 \%, \mathrm{P}=0.156$; Table S1). For those with remnant liver hypoperfusion on POW1, the hypoperfusion area significantly decreased on POW4 [from $11.6(5.5,29.8)$ to $1.1(0,5.8) \mathrm{mL}, \mathrm{P}<0.001$; Figure $\mathrm{S} 2]$.

\section{Association between remnant liver hypoperfusion and vascular injuries}

In the preoperative $3 \mathrm{D}$ reconstruction, eRLI (i.e., liver parenchyma without portal vein support) was found in 52 (51\%) patients by determining the ligated portal veins; the median volume percentage of eRLI/SLV was $4.5 \%$ (1.6\%, $10.6 \%$ ). eRLC (i.e., liver parenchyma without hepatic vein support) was found in 60 (59\%) patients by determining the ligated hepatic veins; the median volume percentage of eRLC/SLV was $7.5 \%(2.7 \%, 12.3 \%)$ (Table 1). Compared with patients without remnant liver hypoperfusion, those with remnant liver hypoperfusion were associated with more presence of eRLC (79\% vs. $42 \%, \mathrm{P}<0.001)$, but not eRLI ( $53 \%$ vs. $49 \%, \mathrm{P}=0.680$ ). Furthermore, eRLC/SLV had better diagnostic significance than eRLI/SLV in predicting remnant liver hypoperfusion (area under ROC curve: 0.745 vs. $0.569, \mathrm{P}=0.026$ by Delong's test; Figure 3). Therefore, remnant liver hypoperfusion detected on postoperative CT scans was associated with hepatic vein related congestion rather than portal vein related ischemia in this cohort of patients.

\section{Association between estimated RLI or RLC and postoperative clinical outcomes}

The clinical outcomes associated with RLI or RLC were explored. Univariate analysis showed that LS $>11.75 \mathrm{kPa}(20)$, smaller eFRL/SLV and larger eRLI/SLV were associated with incidence of PHLF (Figure 4). Multivariate analysis showed that LS $>11.75 \mathrm{kPa}$ [odds ratio (OR), 3.370; $95 \%$ confidence interval (CI), 1.120-10.143; $\mathrm{P}=0.031]$, smaller eFRL/SLV (OR, 0.914; 95\% CI, 0.877-0.952; P<0.001), and larger eRLI/SLV (OR, 1.154; 95\% CI, 1.075-1.240; $\mathrm{P}<0.001$ ) were independent risk factors for PHLF (Figure 4).

Univariate analysis showed that lower hemoglobin level and larger eRLC/SLV were associated with incidence of major postoperative complications (Figure 5). The multivariate analysis showed that lower hemoglobin level (OR, 0.672; 95\% CI, 0.487-0.928; $\mathrm{P}=0.016)$ and larger eRLC/SLV (OR, 1.114; 95\% CI, 1.032-1.204; P=0.006) were independent risk factors for major postoperative complications (Figure 5), which was consistent with the above finding that presence of remnant liver hypoperfusion 


\begin{tabular}{|c|c|c|c|c|c|c|c|}
\hline \multirow{2}{*}{ Variables } & \multirow{2}{*}{$\mathbf{N}$} & \multicolumn{4}{|c|}{ Univariate analysis } & \multicolumn{2}{|c|}{ Multivariate analysis } \\
\hline & & \multicolumn{3}{|c|}{ OR $(95 \% \mathrm{CI})$} & \multirow{2}{*}{$\frac{P \text { Value }}{0.429}$} & \multirow[t]{2}{*}{ OR $(95 \% \mathrm{CI})$} & \multirow[t]{2}{*}{$P$ Value } \\
\hline Age, years & 102 & 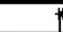 & 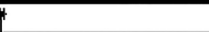 & $1.013(0.981-1.047)$ & & & \\
\hline Sex, female/male & $22 / 80$ & $\longmapsto$ & & $0.942(0.353-2.517)$ & 0.906 & & \\
\hline $\mathrm{ALB}, \geq /<35 \mathrm{~g} / \mathrm{L}$ & $98 / 4$ & & & - & - & & \\
\hline $\mathrm{ALT}, \leq />50 \mathrm{U} / \mathrm{L}$ & $79 / 23$ & $\longmapsto$ & $\longrightarrow$ & $1.238(0.475-3.226)$ & 0.662 & & \\
\hline $\mathrm{AST}, \leq />40 \mathrm{U} / \mathrm{L}$ & $63 / 39$ & & & $2.143(0.931-4.933)$ & 0.073 & & \\
\hline $\mathrm{PT}, \mathrm{s}$ & 102 & & $\longrightarrow$ & $1.551(0.976-2.465)$ & 0.063 & & \\
\hline $\mathrm{HB}$, per $10 \mathrm{~g} / \mathrm{L}$ & 102 & $1=$ & & $0.872(0.703-1.082)$ & 0.214 & & \\
\hline $\mathrm{PLT}, \geq /<100 * 10^{9} / \mathrm{L}$ & $92 / 10$ & $\longmapsto$ & & $1.968(0.529-7.313)$ & 0.312 & & \\
\hline $\mathrm{WBC}, * 10^{9} / \mathrm{L}$ & 102 & & -1 & $1.079(0.935-1.246)$ & 0.298 & & \\
\hline $\mathrm{HBsAg},-/+$ & $44 / 58$ & $\mapsto$ & $=$ & $1.567(0.680-3.609)$ & 0.291 & & \\
\hline Hilar occlusion, $\leq>>20 \mathrm{~min}$ & $54 / 48$ & $\longmapsto$ & $=$ & $1.200(0.532-2.707)$ & 0.660 & & \\
\hline Blood lose,$\leq>400 \mathrm{~mL}$ & $64 / 38$ & $\longmapsto$ & $\longrightarrow$ & $1.114(0.482-2.572)$ & 0.801 & & \\
\hline eFRL/SLV, \% & 102 & 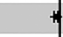 & & $0.943(0.915-0.972)$ & $<0.001$ & $0.914(0.877-0.952)$ & $<0.001$ \\
\hline eRLI/SLV, \% & 102 & & $\Rightarrow$ & $1.078(1.007-1.154)$ & 0.032 & $1.154(1.075-1.240)$ & $<0.001$ \\
\hline eRLC/SLV, \% & 102 & , & 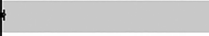 & $0.995(0.945-1.048)$ & 0.849 & & \\
\hline
\end{tabular}

Figure 4 Forest map of univariate and multivariate logistic analysis of risk factors for PHLF. PHLF, post-hepatectomy liver failure; TB, total bilirubin; ALB, albumin; ALT, alanine aminotransferase; AST, aspartate aminotransferase; GGT, $\gamma$-glutamyl transpeptidase; PA, prealbumin; PT, prothrombin time; HB, hemoglobin; PLT, platelet count; WBC, white blood cell; HBsAg, hepatitis B s antigen; HBeAg, hepatitis B e antigen; LS, liver stiffness; CSPH, clinical significant portal hypertension; SLV, standardized liver volume; eFRL, estimated future remnant liver volume; eRLI, estimated portal-vein-associated remnant liver ischemia; eRLC, estimated hepatic-vein-associated remnant liver congestion.

associated with postoperative complications.

\section{Discussion}

The present study demonstrated that postoperative RLI/ RLC can be predicted by preoperative surgical plan using 3DVT, which can be attributed to injury of portal or hepatic veins. It also showed portal vein related RLI was associated with PHLF, and hepatic vein related RLC was associated with major postoperative complications.

In the present study, remnant liver hypoperfusion was detected in $46 \%$ patients on the POW1 CT scans. Previous studies have reported that liver hypoperfusion occurred in a range from $25.3 \%$ to $60.9 \%$ of patients after hepatectomy (6-8), and the incidence of liver hypoperfusion was not significantly different between patients who underwent anatomical or non-anatomical hepatectomy or between patients who underwent major or minor liver resection (7), which suggests liver hypoperfusion is more likely an inevitable result because of the viability of blood vessels and irregularity of segment boundaries (5). Furthermore, the transection plane was usually determined by the demarcation on liver surface after ligation of the corresponding portal vein, while the intrahepatic boundary is not clear. The transection plane may not correspond exactly to the intersegmental boundary in many cases. Some studies have shown injection of fluorescence dye into the corresponding portal vein may help to visualize the boundary $(4,15)$, but it was also revealed that the perfusion area of the portal vein and the drainage area of the hepatic vein in the same segment did not always coincide (21-23). The present study suggests that remnant liver hypoperfusion can be predicted during the preoperative surgery plan, which implies that RLI/RLC may be avoidable or decreased when the transection plane is carefully planned. However, if vessel injuries happen during surgery, more attention should be paid on the postoperative observation and management.

The present study also revealed for the first time that the outcomes of RLI/RLC associated with inflow or outflow vessel injuries were different. Postoperative CT scan cannot distinguish outflow-injured congestion from inflow-injured ischemia in liver parenchyma because hepatic vein occlusion induces localized intrahepatic venous congestion, which has been shown to produce a confusing radiologic image 


\begin{tabular}{|c|c|c|c|c|c|c|c|}
\hline \multirow{2}{*}{ Variables } & \multirow{2}{*}{$\mathbf{N}$} & \multicolumn{4}{|c|}{ Univariate analysis } & \multicolumn{2}{|c|}{ Multivariate analysis } \\
\hline & & \multicolumn{3}{|c|}{ OR $(95 \% \mathrm{CI})$} & \multirow{2}{*}{$\frac{P \text { Value }}{0.178}$} & \multirow[t]{2}{*}{$\mathrm{OR}(95 \% \mathrm{CI})$} & \multirow[t]{2}{*}{$P$ Value } \\
\hline Age, years & 102 & & $H$ & $1.032(0.986-1.081)$ & & & \\
\hline Sex, female/male & $22 / 80$ & $\longmapsto$ & & $1.940(0.404-9.329)$ & 0.408 & & \\
\hline $\mathrm{ALB}, \geq /<35 \mathrm{~g} / \mathrm{L}$ & $98 / 4$ & & 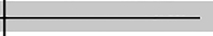 & $6.538(0.846-$ & 0.072 & & \\
\hline $\mathrm{ALT}, \leq>50 \mathrm{U} / \mathrm{L}$ & $79 / 23$ & $\longmapsto$ & 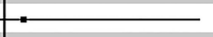 & $1.301(0.372-4.553)$ & 0.680 & & \\
\hline $\mathrm{AST}, \leq />40 \mathrm{U} / \mathrm{L}$ & $63 / 39$ & $\longmapsto$ & $\longrightarrow$ & $0.779(0.245-2.478)$ & 0.673 & & \\
\hline PT, s & 102 & 它 & $\longrightarrow$ & $1.520(0.874-2.645)$ & 0.138 & & \\
\hline $\mathrm{HB}$, per $10 \mathrm{~g} / \mathrm{L}$ & 102 & $1=-1$ & & $0.695(0.522-0.926)$ & 0.013 & $0.672(0.487-0.928)$ & 0.016 \\
\hline PLT, $\geq k<100 * 10^{9} / \mathrm{L}$ & $92 / 10$ & $\longmapsto$ & $\rightarrow$ & $1.519(0.290-7.965)$ & 0.621 & & \\
\hline $\mathrm{WBC}, * 10^{9} / \mathrm{L}$ & 102 & & -1 & $1.189(0.977-1.449)$ & 0.085 & & \\
\hline $\mathrm{HBsAg},-/+$ & $44 / 58$ & $\longmapsto$ & $=$ & $1.163(0.381-3.553)$ & 0.791 & & \\
\hline Hilar occlusion, $\leq />20 \mathrm{~min}$ & $54 / 48$ & $\longmapsto$ & - & $1.343(0.448-4.028)$ & 0.599 & & \\
\hline Blood lose,$\leq>400 \mathrm{~mL}$ & $64 / 38$ & $\longmapsto$ & $\rightarrow$ & $1.581(0.523-4.773)$ & 0.417 & & \\
\hline eFRL/SLV, \% & 102 & & + & $0.996(0.967-1.025)$ & 0.762 & & \\
\hline eRLI/SLV, \% & 102 & & 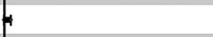 & $1.044(0.989-1.102)$ & 0.117 & & \\
\hline eRLC/SLV, \% & 102 & & $\rightarrow 1$ & $1.108(1.030-1.192)$ & 0.006 & $1.114(1.032-1.204)$ & 0.006 \\
\hline
\end{tabular}

Figure 5 Forest map of univariate and multivariate logistic analysis of risk factors for major postoperative complications. TB, total bilirubin; ALB, albumin; ALT, alanine aminotransferase; AST, aspartate aminotransferase; GGT, $\gamma$-glutamyl transpeptidase; PA, pre-albumin; PT, prothrombin time; HB, hemoglobin; PLT, platelet count; WBC, white blood cell; HBsAg, hepatitis B s antigen; HBeAg, hepatitis B e antigen; LS, liver stiffness; CSPH, clinical significant portal hypertension; SLV, standardized liver volume; eFRL, estimated future remnant liver volume; eRLI, estimated portal-vein-associated remnant liver ischemia; eRLC, estimated hepatic-vein-associated remnant liver congestion.

mimicking inflow insufficiency $(9,10)$. With the help of 3DVT, we found $51 \%$ and $59 \%$ patients may develop RLI or RLC respectively, while remnant liver hypoperfusion detected on POW1 CT scan associated with hepatic vein injury related RLC, rather than portal vein injury related RLI. This may be explained by the fact that the transection plane was usually determined by the demarcation on the liver surface after ligation of the portal vein branches, instead of hepatic vein branches. This raises an importance of defining the hepatic vein-associated boundary segments given that the portal vein-supplied territory does not match the hepatic vein-supplied territory in some cases (21-23).

PHLF has a subacute course, and an inadequate functional reserve of the remnant liver is central in its etiology (24). The present study showed that LS $>11.75 \mathrm{kPa}$ and lower eFRL/SLV were independent risk factors for PHLF, indicating that inadequate functional reserve and remnant liver volume were the main reasons for PHLF. Our previous study demonstrated that LS measured by shear wave elastography higher than $11.75 \mathrm{kPa}$ was associated with a higher risk of PHLF, which was also demonstrated in the present study (20). The interesting finding was that
eRLI/SLV independently predicted PHLF, while eRLC/ SLV independently predicted postoperative complications, especially effusions requiring additional percutaneous drainage and antibiotic treatments, which was consistent with previous studies. Gertsch et al. reported that patients with RLI had a higher risk of biliary leak and higher peak levels of postoperative AST and ALT (6). Thus, improper reserve of remnant liver with inflow or outflow injuries would increase risk of poor clinical outcomes. PVE and ALPPS should be applied to increase future remnant liver instead of preservation of RLI/RLC area.

The major limitation of this study lies in the inevitable difference between the actual transection plane and the estimated one on the $3 \mathrm{D}$ model. The deviation of the transection line would result in a miscalculation of the estimated RLI/RLC area. We have tested the consistency between predicted resected liver volume and actual resected liver volume (Figure S1). A significant correlation existed between the predicted liver resection volume and the volume of the resected liver measured by Archimedes water-displacement method, supporting that our system enabled the accurate prediction of the liver transection 
plane. Nevertheless, the discordance may still exist between radiologist and surgeons because there is no anatomical landmark within the liver, so visualization of liver segments by indocyanine green retention may help us to minimize the discordance.

Only considering blood supply from portal vein in this study is because of the limit in $3 \mathrm{D}$ reconstruction technique. However, hepatic artery is an additional source of blood supply to liver parenchyma, which may contribute to development of RLI. Not considering arterial supply may lead to a miscalculation of estimated RLI.

\section{Conclusions}

The present study demonstrated that remnant liver hypoperfusion can be predicted during the preoperative surgical plan by 3 DVT. Portal vein related RLI was associated with PHLF, and hepatic vein related RLC was associated with major postoperative complications. The present study suggested that preservation of the hepatic vein and complete removal of the perfusion territory of ligated vessels are essential procedures to reduce RLI/RLC and the risk of PHLF or other surgical complications.

\section{Acknowledgments}

Part of the manuscript has been presented as a poster presentation (Abstract ID: E10183) in 10th Asia-Pacific Primary Liver Cancer Expert Congress (APPLE 2019).

Funding: This work was supported by grants from the Leading Investigator Program of Shanghai municipal government (17XD1401100), the National Key Basic Research Program (973 Program; 2015CB554005) from the Ministry of Science and Technology of China, and the National Natural Science Foundation of China (81372655, 81472224 and 81672326).

\section{Footnote}

Reporting Checklist: The authors have completed the STARD reporting checklist. Available at http://dx.doi.org/10.21037/ atm-20-7920

Data Sharing Statement: Available at http://dx.doi. org/10.21037/atm-20-7920

Peer Review File: Available at http://dx.doi.org/10.21037/ atm-20-7920
Conflicts of Interest: All authors have completed the ICMJE uniform disclosure form (available at http://dx.doi. org/10.21037/atm-20-7920). The authors have no conflicts of interest to declare.

Ethical Statement: The authors are accountable for all aspects of the work in ensuring that questions related to the accuracy or integrity of any part of the work are appropriately investigated and resolved. The study was conducted in accordance with the Declaration of Helsinki (as revised in 2013). The study was approved by institutional ethics board of Zhongshan Hospital, Fudan University (No. B2018-254) and informed consent was taken from all individual participants.

Open Access Statement: This is an Open Access article distributed in accordance with the Creative Commons Attribution-NonCommercial-NoDerivs 4.0 International License (CC BY-NC-ND 4.0), which permits the noncommercial replication and distribution of the article with the strict proviso that no changes or edits are made and the original work is properly cited (including links to both the formal publication through the relevant DOI and the license). See: https://creativecommons.org/licenses/by-nc-nd/4.0/.

\section{References}

1. Mullen JT, Ribero D, Reddy SK, et al. Hepatic insufficiency and mortality in 1,059 noncirrhotic patients undergoing major hepatectomy. J Am Coll Surg 2007;204:854-62; discussion 862-4.

2. Balzan S, Belghiti J, Farges O, et al. The "50-50 criteria" on postoperative day 5: an accurate predictor of liver failure and death after hepatectomy. Ann Surg 2005;242:824-8; discussion 828-9.

3. Rahbari NN, Garden OJ, Padbury R, et al. Posthepatectomy liver failure: a definition and grading by the International Study Group of Liver Surgery (ISGLS). Surgery 2011;149:713-24.

4. Makuuchi M, Hasegawa H, Yamazaki S. Ultrasonically guided subsegmentectomy. Surg Gynecol Obstet 1985;161:346-50.

5. Shindoh J, Mise Y, Satou S, et al. The intersegmental plane of the liver is not always flat--tricks for anatomical liver resection. Ann Surg 2010;251:917-22.

6. Gertsch P, Vandoni RE, Pelloni A, et al. Localized hepatic ischemia after liver resection: a prospective evaluation. Ann Surg 2007;246:958-64; discussion 964-5. 
7. Cho JY, Han HS, Choi Y, et al. Association of remnant liver ischemia with early recurrence and poor survival after liver resection in patients with hepatocellular carcinoma. JAMA Surg 2017;152:386-92.

8. Yamashita S, Venkatesan AM, Mizuno T, et al. Remnant liver ischemia as a prognostic factor for cancer-specific survival after resection of colorectal liver metastases. JAMA Surg 2017;152:e172986.

9. Hwang S, Lee SG, Park KM, et al. Hepatic venous congestion in living donor liver transplantation: preoperative quantitative prediction and follow-up using computed tomography. Liver Transpl 2004;10:763-70.

10. Hwang S, Lee SG, Ha TY, et al. Feasibility assessment for tailoring preservation of segment VIII hepatic vein during left liver graft procurement. Hepatogastroenterology 2006;53:928-32.

11. Saito S, Yamanaka J, Miura K, et al. A novel 3D hepatectomy simulation based on liver circulation: application to liver resection and transplantation. Hepatology 2005;41:1297-304.

12. Simpson AL, Geller DA, Hemming AW, et al. Liver planning software accurately predicts postoperative liver volume and measures early regeneration. J Am Coll Surg 2014;219:199-207.

13. Li PP, Wang ZH, Huang G, et al. Application of liver three-dimensional visualization technologies in the treatment planning of hepatic malignant tumor. Zhonghua Wai Ke Za Zhi 2017;55:916-22.

14. Mise Y, Hasegawa K, Satou S, et al. How has virtual hepatectomy changed the practice of liver surgery? Ann Surg 2018;268:127-33.

15. Takamoto T, Hashimoto T, Ogata S, et al. Planning of anatomical liver segmentectomy and subsegmentectomy with 3-dimensional simulation software. Am J Surg

Cite this article as: $\mathrm{Li} \mathrm{XL}, \mathrm{Xu} B$, Zhu XD, Huang C, Shi GM, Shen YH, Wu D, Tang M, Tang ZY, Zhou J, Fan J, Sun HC. Simulation of portal/hepatic vein associated remnant liver ischemia/congestion by three-dimensional visualization technology based on preoperative CT scan. Ann Transl Med 2021;9(9):756. doi: 10.21037/atm-20-7920
2013;206:530-8.

16. Huang C, Zhu XD, Shi GM, et al. Dexamethasone for postoperative hyperbilirubinemia in patients after liver resection: an open-label, randomized controlled trial. Surgery 2019;165:534-40.

17. Dindo D, Demartines N, Clavien PA. Classification of surgical complications: a new proposal with evaluation in a cohort of 6336 patients and results of a survey. Ann Surg 2004;240:205-13.

18. Urata K, Kawasaki S, Matsunami H, et al. Calculation of child and adult standard liver volume for liver transplantation. Hepatology 1995;21:1317-21.

19. Mise Y, Aloia TA, Vauthey JN. Liver volumetry in virtual hepatectomy must account for vascular territories. J Am Coll Surg 2014;219:1194-5.

20. Shen Y, Zhou C, Zhu G, et al. Liver stiffness assessed by shear wave elastography predicts postoperative liver failure in patients with hepatocellular carcinoma. J Gastrointest Surg 2017;21:1471-9.

21. Mise Y, Hasegawa K, Satou S, et al. Venous reconstruction based on virtual liver resection to avoid congestion in the liver remnant. Br J Surg 2011;98:1742-51.

22. Sato F, Igami T, Ebata T, et al. A study of the right intersectional plane (right portal scissura) of the liver based on virtual left hepatic trisectionectomy. World J Surg 2014;38:3181-5.

23. Abe H, Yamazaki S, Moriguchi M, et al. Perfusion and drainage difference in the liver parenchyma: Regional plane in segment 6. Biosci Trends 2017;11:326-32.

24. van den Broek MA, Olde DS, Dejong CH, et al. Liver failure after partial hepatic resection: definition, pathophysiology, risk factors and treatment. Liver Int 2008;28:767-80. 


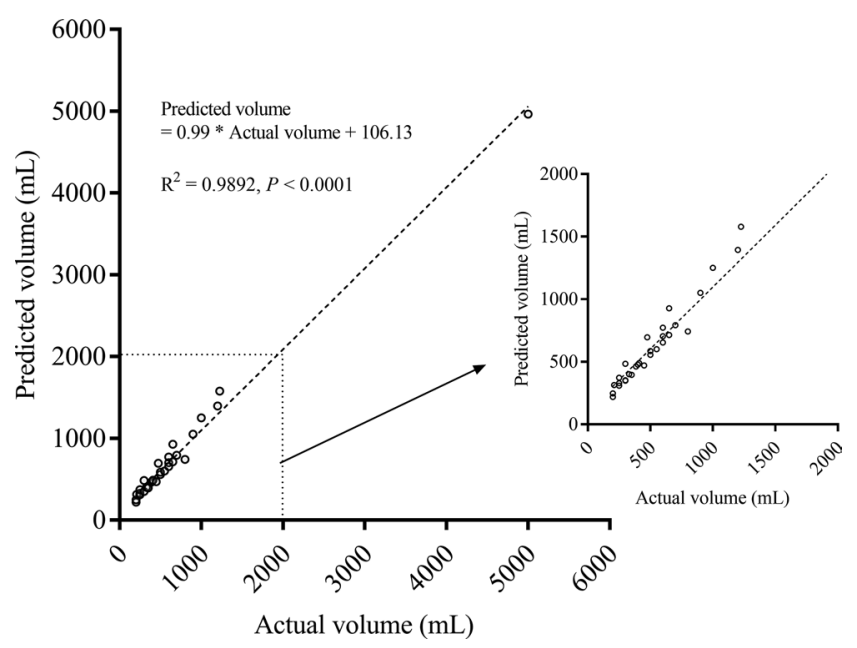

Figure S1 Correlation between predicted liver resection volume and actual volume of resected specimen measured by Archimedes drainage method. A significant correlation existed between the predicted liver resection volume and the actual volume of the resected specimen $\left(\mathrm{R}^{2}=0.989, \mathrm{P}<0.0001\right)$. 
Table S1 Comparison of baseline characteristics, surgical procedure, liver volumetry, estimated RLI/RLC areas and clinical outcomes between patients with and without remnant liver hypoperfusion detected in postoperative CT scan (POW1)

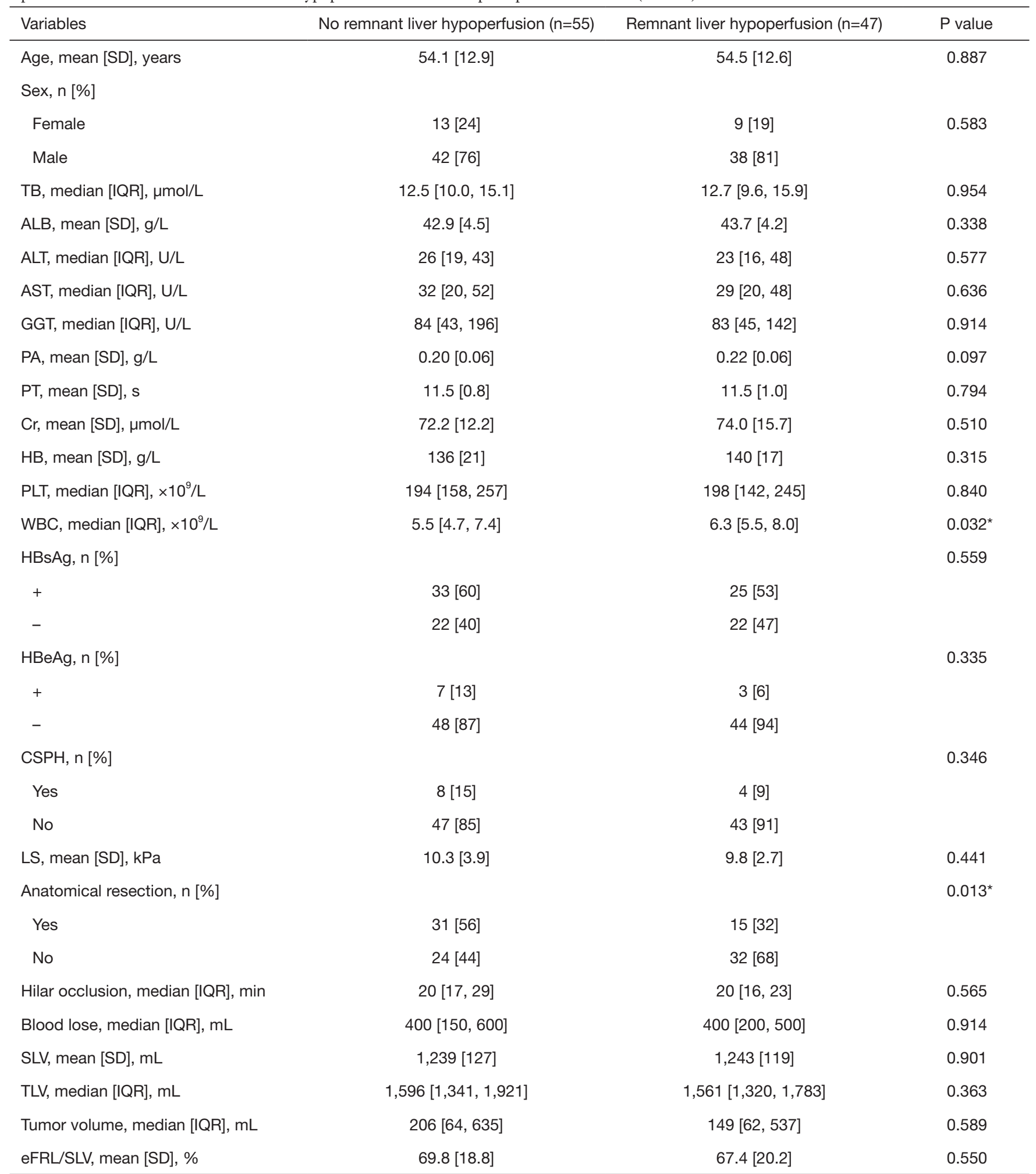

Table S1 (continued) 
Table S1 (continued)

\begin{tabular}{|c|c|c|c|}
\hline Variables & No remnant liver hypoperfusion $(n=55)$ & Remnant liver hypoperfusion $(n=47)$ & $P$ value \\
\hline eRLI, n [\%] & & & 0.680 \\
\hline Absent & $28[51]$ & $22[47]$ & \\
\hline Present & 27 [49] & $25[53]$ & \\
\hline eRLI/SLV, median [IQR], \% & $2.6[0.9,7.5]$ & $6.2[2.7,17.0]$ & $0.023^{*}$ \\
\hline Absent & $32[58]$ & $10[21]$ & \\
\hline Present & $23[42]$ & 37 [79] & \\
\hline eRLC/SLV, median [IQR], \% & $3.2[1.3,9.7]$ & $8.7[4.5,12.9]$ & $0.018^{*}$ \\
\hline PHLF, [\%] & & & 0.156 \\
\hline Major & $4[7]$ & $11[23]$ & \\
\hline Minor or none & 51 [93] & $36[77]$ & \\
\hline
\end{tabular}

*, P<0.05. CT, computed tomography; POW, postoperative week; HCC, hepatocellular carcinoma; ICC, intrahepatic cholangiocarcinoma; TB, total bilirubin; ALB, albumin; ALT, alanine aminotransferase; AST, aspartate aminotransferase; GGT, $\gamma$-glutamyl transpeptidase; PA, pre-albumin; INR, international normalized ratio; Cr, creatinine; HB, hemoglobin; PLT, platelet count; WBC, white blood cell; HBsAg, hepatitis B s antigen; HBeAg, hepatitis B e antigen; CSPH, clinical significant portal hypertension; LS, liver stiffness; SLV, standardized liver volume; TLV, total liver volume; eFRL, estimated future remnant liver volume; eRLI, estimated portal-vein-associated remnant liver ischemia; eRLC, estimated hepatic-vein-associated remnant liver congestion; PHLF, post-hepatectomy liver failure.

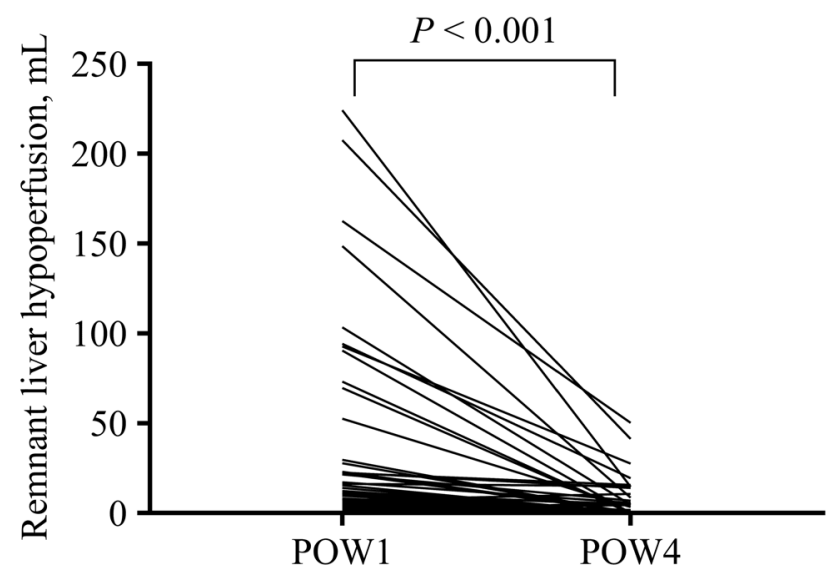

Figure S2 The change of volume of remnant liver hypoperfusion from POW1 to POW4. The remnant liver hypoperfusion area significantly decreased from $11.6(5.5,29.8) \mathrm{mL}$ on POW1 to $1.1(0,5.8) \mathrm{mL}$ on POW4 $(\mathrm{P}<0.001)$. POW, postoperative week. 